Research Article

\title{
Extended Jacobi Functions via Riemann-Liouville Fractional Derivative
}

\section{Bayram Çekim and Esra Erkuş-Duman}

Gazi University, Faculty of Science, Department of Mathematics, Teknikokullar, 06500 Ankara, Turkey

Correspondence should be addressed to Esra Erkuş-Duman; eduman@gazi.edu.tr

Received 19 January 2013; Accepted 3 April 2013

Academic Editor: Mohamed Kamal Aouf

Copyright (C) 2013 B. Cekim and E. Erkuş-Duman. This is an open access article distributed under the Creative Commons Attribution License, which permits unrestricted use, distribution, and reproduction in any medium, provided the original work is properly cited.

By means of the Riemann-Liouville fractional calculus, extended Jacobi functions are defined and some of their properties are obtained. Then, we compare some properties of the extended Jacobi functions extended Jacobi polynomials. Also, we derive fractional differential equation of generalized extended Jacobi functions.

\section{Introduction}

Fractional calculus is examined by many mathematicians such as Liouville, Riemann, and Caputo. In recent years, the theory of fractional calculus, integrals, and derivatives of fractional orders has become an active research area in mathematical analysis. This theory has also been applied for many fundamental areas such as biology, physics, electrochemistry, economics, probability theory, and statistics $[1,2]$.

In this paper, we use fractional calculus in the theory of special functions. More precisely, we study the extended Jacobi function via the Riemann-Liouville (fractional) operator. Furthermore, we define a generalized extended Jacobi function which is a solution of the fractional differential equation.

Throughout the paper, we consider the RiemannLiouville fractional derivative of a function $f$ with order $\mu$ defined by

$$
D^{\mu} f(t):=D^{m}\left[J^{m-\mu} f(t)\right]
$$

where $m \in \mathbb{N}, m-1 \leq \mu<m$, and

$$
J^{m-\mu} f(t):=\frac{1}{\Gamma(m-\mu)} \int_{0}^{t}(t-\tau)^{m-\mu-1} f(\tau) d \tau
$$

is the Riemann-Liouville fractional integral of $f$ with order $m-\mu$. Here, $\Gamma$ denotes the classical gamma function. It is easy to see that the fractional derivative of the power function $f(t)=t^{\alpha}$ is given by

$$
D^{\mu} t^{\alpha}=\frac{\Gamma(\alpha+1)}{\Gamma(\alpha-\mu+1)} t^{\alpha-\mu},
$$

where $\alpha \geq-1, \mu \geq 0$, and $t>0$. We know from [3] that if $f$ is a continuous function in $[0, t]$ and $\varphi$ has $n+1$ continuous derivatives in $[0, t]$, then the fractional derivative of the product $\varphi f$, that is, the Leibniz rule, is given as follows:

$$
D^{\mu}[\varphi(t) f(t)]=\sum_{k=0}^{\infty}\left(\begin{array}{c}
\mu \\
k
\end{array}\right) \varphi^{(k)}(t) D^{\mu-k} f(t) .
$$

Furthermore, according to Jumarie [4], if both of the functions $f(x)$ and $x(t)$ from $\mathbb{R}$ into itself have derivatives of order $\alpha, 0<\alpha<1$, one has the chain derivative rule for fractional calculus:

$$
\frac{d^{\alpha}}{d t^{\alpha}} f(x(t))=\frac{d^{\alpha} f(x)}{d x^{\alpha}}\left(\frac{d x}{d t}\right)^{\alpha} .
$$

It is well known that the classical Gauss differential equation is given as follows:

$$
x(1-x) y^{\prime \prime}+[c-(a+b+1) x] y^{\prime}-a b y=0 .
$$


As usual, (6) has a solution of the hypergeometric function defined by

$$
F(a, b ; c ; \mathrm{x})=\sum_{k=0}^{\infty} \frac{(a)_{k}(b)_{k}}{(c)_{k} k !} x^{k} ; \quad|x|<1,
$$

where $(\lambda)_{k}$ is the Pochhammer symbol

$$
(\lambda)_{k}=\lambda(\lambda+1) \cdots(\lambda+k-1), \quad(\lambda)_{0}=1
$$

Furthermore, we have the following transformation for the hypergeometric functions

$$
F(a, b ; c ; x)=(1-x)^{-a} F\left(a, c-b ; c ; \frac{-x}{1-x}\right),
$$

where $|-x /(1-x)|<1,|x|<1$.

Fujiwara [5] studied the polynomial $F_{n}^{(\alpha, \beta)}(x ; a, b, c)$ which is called the extended Jacobi polynomial (EJP) and defined by the Rodrigues formula

$$
\begin{aligned}
F_{n}^{(\alpha, \beta)}(x ; a, b, c)= & \frac{(-c)^{n}}{n !}(x-a)^{-\alpha}(b-x)^{-\beta} \\
& \times \frac{d^{n}}{d x^{n}}\left\{(x-a)^{n+\alpha}(b-x)^{n+\beta}\right\},
\end{aligned}
$$

where $\alpha, \beta>-1$ and $c>0$. The EJPs $F_{n}^{(\alpha, \beta)}(x ; a, b, c)$ are orthogonal over the interval $(a, b)$ with respect to the weight function $\omega(x ; a, b)=(x-a)^{\alpha}(b-x)^{\beta}$. In fact, it is hold that

$$
\begin{aligned}
& \int_{a}^{b}(x-a)^{\alpha}(b-x)^{\beta} F_{n}^{(\alpha, \beta)}(x ; a, b, c) F_{m}^{(\alpha, \beta)}(x ; a, b, c) d x \\
& =\left(\left(c^{m+n}(-1)^{\alpha+\beta+1}(a-b)^{m+n+\alpha+\beta+1}\right.\right. \\
& \quad \times \Gamma(\alpha+n+1) \Gamma(\beta+n+1)) \\
& \left.\quad \times(n !(\alpha+\beta+2 n+1) \Gamma(\alpha+\beta+n+1))^{-1}\right) \delta_{m, n},
\end{aligned}
$$

where $\delta_{m, n}$ is the Kronecker delta and $\min \{\mathbb{R}(\alpha), \mathbb{R}(\beta)\}>$ $-1 ; m, n \in \mathbb{N}_{0}:=\mathbb{N} \cup\{0\}$.

\section{Extended Jacobi Functions (EJFs)}

In this section, we define the extended Jacobi functions (EJFs) and obtain their some significant properties.

Definition 1. Assume that $\alpha, \beta>-1$ and $c>0$. The extended Jacobi functions are defined to be as the following Rodrigues formula:

$$
\begin{aligned}
F_{\nu}^{(\alpha, \beta)}(x ; a, b, c)= & \frac{(-c)^{\nu}}{\Gamma(\nu+1)}(x-a)^{-\alpha}(b-x)^{-\beta} \\
& \times \frac{d^{\nu}}{d x^{\nu}}\left\{(x-a)^{\nu+\alpha}(b-x)^{\nu+\beta}\right\},
\end{aligned}
$$

where $v>0$ and $d^{\nu} / d x^{v}$ is the Riemann-Liouville fractional differentiation operator.
Theorem 2. The explicit form of the EJFs is given by

$$
F_{v}^{(\alpha, \beta)}(x ; a, b, c)=c^{\nu} \sum_{k=0}^{\infty}\left(\begin{array}{c}
\nu+\alpha \\
v-k
\end{array}\right)\left(\begin{array}{c}
\nu+\beta \\
k
\end{array}\right)(x-a)^{k}(x-b)^{\nu-k}
$$

where $\alpha, \beta>-1$ and $c>0$.

Proof. If we use the Leibniz rule (4) in (12), then we have

$$
\begin{aligned}
F_{\nu}^{(\alpha, \beta)}(x ; a, b, c) & \\
= & \frac{(-c)^{\nu}}{\Gamma(\nu+1)}(x-a)^{-\alpha}(b-x)^{-\beta} \\
& \times \sum_{k=0}^{\infty}\left(\begin{array}{l}
\nu \\
k
\end{array}\right)\left\{D_{x}^{k}\left[(b-x)^{\beta+\nu}\right]\right\}\left\{D_{x}^{\nu-k}\left[(x-a)^{\alpha+\nu}\right]\right\} .
\end{aligned}
$$

It follows from the definition of fractional derivative that

$$
\begin{aligned}
D_{x}^{\nu-k}\left[(x-a)^{\alpha+\nu}\right] & \\
& =\sum_{r=0}^{\infty}\left(\begin{array}{c}
\nu+\alpha \\
r
\end{array}\right) a^{r}(-1)^{r} \frac{\Gamma(\nu+\alpha-r+1)}{\Gamma(k+\alpha-r+1)} x^{k+\alpha-r} .
\end{aligned}
$$

From (15) and (14), we get that

$$
\begin{aligned}
F_{\nu}^{(\alpha, \beta)}(x ; a, b, c)= & \frac{(-c)^{\nu}}{\Gamma(\nu+1)}(x-a)^{-\alpha}(b-x)^{-\beta} \\
& \times \sum_{k=0}^{\infty}\left(\begin{array}{l}
\nu \\
k
\end{array}\right)(-1)^{k} \frac{\Gamma(\beta+\nu+1)}{\Gamma(\beta+\nu-k+1)} \\
& \times(b-x)^{\beta+(\nu-k)} \\
& \times \sum_{r=0}^{\infty}\left(\begin{array}{c}
\nu+\alpha \\
r
\end{array}\right) a^{r}(-1)^{r} \frac{\Gamma(\nu+\alpha-r+1)}{\Gamma(k+\alpha-r+1)} \\
& \times x^{k+\alpha-r} .
\end{aligned}
$$

Using the fact that

$$
\sum_{r=0}^{\infty} \frac{\Gamma(\alpha+k+1)}{\Gamma(k+\alpha-r+1) r !} a^{r}(-1)^{r} x^{\alpha+k-r}=(x-a)^{\alpha+k},
$$

the proof is completed.

Corollary 3. The another explicit form of the EJFs is given by

$$
\begin{aligned}
F_{v}^{(\alpha, \beta)}(x ; a, b, c)= & c^{\nu} \sum_{k=0}^{\infty}(-1)^{k}\left(\begin{array}{c}
\nu+\alpha \\
k
\end{array}\right) \\
& \times\left(\begin{array}{c}
\nu+\beta \\
\nu-k
\end{array}\right)(x-a)^{\nu-k}(x-b)^{k},
\end{aligned}
$$

where $\alpha, \beta>-1$ and $c>0$. 
Proof. This formula can be proved similar to Theorem 2 by taking the following:

$$
\begin{aligned}
& F_{\nu}^{(\alpha, \beta)}(x ; a, b, c) \\
& =\frac{(-c)^{\nu}}{\Gamma(\nu+1)}(x-a)^{-\alpha}(b-x)^{-\beta} \\
& \quad \times \sum_{k=0}^{\infty}\left(\begin{array}{l}
\nu \\
k
\end{array}\right)\left\{D_{x}^{k}\left[(x-a)^{\alpha+\nu}\right]\right\}\left\{D_{x}^{\nu-k}\left[(b-x)^{\beta+\nu}\right]\right\} .
\end{aligned}
$$

Remark 4. If we get $a=1, b=-1$, and $c=1 / 2$ in (18), then (18) is reduced to the explicit formula satisfied by the $g-$ Jacobi functions in [6].

Theorem 5. The extended Jacobi functions have the following representation:

$$
\begin{aligned}
F_{v}^{(\alpha, \beta)}(x ; a, b, c)= & c^{\nu}(a-b)^{\nu}\left(\begin{array}{c}
\nu+\alpha \\
v
\end{array}\right) \\
& \times F\left(-v, \alpha+\beta+\nu+1 ; \alpha+1 ; \frac{a-x}{a-b}\right),
\end{aligned}
$$

where $F$ is the hypergeometric function defined in (7).

Proof. Writing $(x-a)+(a-b)$ instead of $(x-b)$ in (13) and using binomial expansion, we obtain

$$
\begin{aligned}
& F_{v}^{(\alpha, \beta)}(x ; a, b, c) \\
& =c^{\nu} \sum_{k=0}^{\infty}\left(\begin{array}{c}
\nu+\alpha \\
v-k
\end{array}\right)\left(\begin{array}{c}
\nu+\beta \\
k
\end{array}\right)(x-a)^{k}(x-a+a-b)^{\nu-k} \\
& =c^{\nu} \sum_{k=0}^{\infty}\left(\begin{array}{l}
\nu+\alpha \\
v-k
\end{array}\right)\left(\begin{array}{c}
\nu+\beta \\
k
\end{array}\right)(x-a)^{k} \\
& \times \sum_{r=0}^{\infty}\left(\begin{array}{c}
v-k \\
r
\end{array}\right)(x-a)^{r}(a-b)^{\nu-k-r} \\
& =c^{\nu} \sum_{r=0}^{\infty} \sum_{k=0}^{r}\left(\begin{array}{l}
\nu+\alpha \\
v-k
\end{array}\right)\left(\begin{array}{c}
\nu+\beta \\
k
\end{array}\right) \\
& \times\left(\begin{array}{l}
\nu-k \\
r-k
\end{array}\right)(x-a)^{r}(a-b)^{\nu-r} \\
& =c^{\nu} \sum_{r=0}^{\infty}(x-a)^{r}(a-b)^{\nu-r} \sum_{k=0}^{r}\left(\begin{array}{c}
\nu+\alpha \\
v-k
\end{array}\right)\left(\begin{array}{c}
\nu+\beta \\
k
\end{array}\right)\left(\begin{array}{c}
\nu-k \\
r-k
\end{array}\right) \\
& =c^{\nu} \sum_{r=0}^{\infty}(x-a)^{r}(a-b)^{\nu-r}\left(\begin{array}{c}
\nu+\alpha \\
\nu-r
\end{array}\right) \sum_{k=0}^{r}\left(\begin{array}{c}
r+\alpha \\
r-k
\end{array}\right)\left(\begin{array}{c}
\nu+\beta \\
k
\end{array}\right) \text {. }
\end{aligned}
$$

Using the following identity:

$$
(1-x)^{\beta+v}(1-x)^{\alpha+r}=(1-x)^{\beta+\alpha+\nu+r},
$$

we have

$$
\left(\begin{array}{c}
\beta+\alpha+\nu+r \\
r
\end{array}\right)=\sum_{k=0}^{r}\left(\begin{array}{c}
r+\alpha \\
r-k
\end{array}\right)\left(\begin{array}{c}
\nu+\beta \\
k
\end{array}\right) .
$$

Substituting (23) in (21), we get that

$$
\begin{aligned}
F_{\nu}^{(\alpha, \beta)} & (x ; a, b, c) \\
& =c^{\nu} \sum_{r=0}^{\infty}(x-a)^{r}(a-b)^{\nu-r}\left(\begin{array}{c}
\nu+\alpha \\
v-r
\end{array}\right)\left(\begin{array}{c}
\beta+\alpha+\nu+r \\
r
\end{array}\right) \\
& =c^{\nu}(a-b)^{\nu}\left(\begin{array}{c}
\nu+\alpha \\
v
\end{array}\right) \sum_{r=0}^{\infty} \frac{(-v)_{r}(\beta+\alpha+\nu+1)_{r}}{r !(\alpha+1)_{r}}\left(\frac{a-x}{a-b}\right)^{r} \\
& =c^{\nu}(a-b)^{\nu}\left(\begin{array}{c}
\nu+\alpha \\
v
\end{array}\right) F\left(-v, \alpha+\beta+\nu+1 ; \alpha+1 ; \frac{a-x}{a-b}\right),
\end{aligned}
$$

which is the desired result.

Remark 6. If we get $a=1, b=-1$, and $c=1 / 2$ in (20), then (20) is reduced to the hypergeometric function representation satisfying the $g-$ Jacobi functions in [6].

Corollary 7. The extended Jacobi functions (EJFs) hold the following hypergeometric function:

$$
\begin{aligned}
F_{\nu}^{(\alpha, \beta)}(x ; a, b, c)= & c^{\nu}(a-b)^{\nu}\left(\begin{array}{c}
\nu+\alpha \\
v
\end{array}\right) \\
& \times\left(\frac{x-b}{a-b}\right)^{\nu} F\left(-v,-\beta-v ; \alpha+1 ; \frac{x-a}{x-b}\right) .
\end{aligned}
$$

Proof. Applying (9) to (20), the proof follows.

Remark 8. Taking $a=1, b=-1$, and $c=1 / 2$ in Corollary 7 , we give the following hypergeometric function representation for the $g$ - Jacobi functions in [6]:

$$
P_{\nu}^{(\alpha, \beta)}(x)=\left(\begin{array}{c}
\nu+\alpha \\
v
\end{array}\right)\left(\frac{x+1}{2}\right)^{\nu} F\left(-v,-\beta-v ; \alpha+1 ; \frac{x-1}{x+1}\right) .
$$

Corollary 9. The extended Jacobi functions can be presented as follows:

$$
\begin{aligned}
F_{\nu}^{(\alpha, \beta)}(x ; a, b, c)= & (-1)^{\nu} c^{\nu}(a-b)^{\nu}\left(\begin{array}{c}
\nu+\beta \\
v
\end{array}\right) \\
& \times F\left(-\nu, \alpha+\beta+\nu+1 ; \beta+1 ; \frac{b-x}{b-a}\right) .
\end{aligned}
$$

Remark 10. Taking $a=1, b=-1$, and $c=1 / 2$ in Corollary 9 , we give the following hypergeometric function representation for the $g-$ Jacobi functions in [6]:

$$
\begin{aligned}
P_{\nu}^{(\alpha, \beta)}(x)= & (-1)^{\nu}\left(\begin{array}{c}
\nu+\beta \\
\nu
\end{array}\right) \\
& \times F\left(-\nu, \alpha+\beta+\nu+1 ; \beta+1 ; \frac{1+x}{2}\right) .
\end{aligned}
$$


Corollary 11. The EJFs hold the following hypergeometric function:

$$
\begin{aligned}
F_{v}^{(\alpha, \beta)}(x ; a, b, c)= & (-1)^{\nu} c^{v}(a-b)^{\nu}\left(\begin{array}{c}
\nu+\beta \\
v
\end{array}\right)\left(\frac{x-a}{b-a}\right)^{v} \\
& \times F\left(-v,-\alpha-v ; \beta+1 ; \frac{x-b}{x-a}\right) .
\end{aligned}
$$

Proof. Using (9) and (27), we complete the proof immediately.

Remark 12. Taking $a=1, b=-1$, and $c=1 / 2$ in Corollary 11, we give the following hypergeometric function representation for the $g-$ Jacobi functions in [6]:

$$
P_{\nu}^{(\alpha, \beta)}(x)=\left(\begin{array}{c}
\nu+\beta \\
\nu
\end{array}\right)\left(\frac{x-1}{2}\right)^{\nu} F\left(-v,-\alpha-v ; \beta+1 ; \frac{x+1}{x-1}\right) .
$$

Theorem 13. For the extended Jacobi functions, one has

$$
\begin{aligned}
(x-b) & \frac{d}{d x} F_{\nu}^{(\alpha, \beta)}(x ; a, b, c) \\
= & \nu F_{v}^{(\alpha, \beta)}(x ; a, b, c) \\
& +c(a-b)(\beta+\nu) F_{\nu-1}^{(\alpha+1, \beta)}(x ; a, b, c), \\
(x-a) & \frac{d}{d x} F_{\nu}^{(\alpha, \beta)}(x ; a, b, c) \\
= & \nu F_{v}^{(\alpha, \beta)}(x ; a, b, c) \\
& -c(a-b)(\alpha+\nu) F_{\nu-1}^{(\alpha, \beta+1)}(x ; a, b, c) .
\end{aligned}
$$

Proof. For the proof of (31), it is enough to use Corollaries 7 and 11 , respectively.

Theorem 14. The extended Jacobi functions hold the differential equation of second-order

$$
\begin{aligned}
(x-a) & (b-x) Y^{\prime \prime}(x) \\
+ & {[b(\alpha+1)+a(\beta+1)-x(\alpha+\beta+2)] } \\
& \times Y^{\prime}(x)+\nu(\alpha+\beta+\nu+1) Y(x)=0
\end{aligned}
$$

or

$$
\begin{aligned}
& \frac{d}{d x}\left[(x-a)^{\alpha+1}(b-x)^{\beta+1} Y^{\prime}(x)\right] \\
& \quad+\nu(\alpha+\beta+\nu+1)(x-a)^{\alpha}(b-x)^{\beta} Y(x)=0 .
\end{aligned}
$$

Proof. With the help of (6) and (7), the hypergeometric function $F(-\nu, \nu+\alpha+\beta+1 ; \alpha+1 ; t)$ satisfies

$$
t(1-t) F^{\prime \prime}+[\alpha+1-t(\alpha+\beta+2)] F^{\prime}+\nu(\alpha+\beta+\nu+1) F=0
$$

where $0 \leq|t|<1$. Writing $(a-x) /(a-b)$ instead of $t$ in the last, we get

$$
\begin{gathered}
(x-a)(b-x) Y^{\prime \prime}(x)+[b(\alpha+1)+a(\beta+1)-x(\alpha+\beta+2)] \\
\times Y^{\prime}(x)+v(\alpha+\beta+v+1) Y(x)=0 .
\end{gathered}
$$

Thus, the extended Jacobi functions having the hypergeometric function (20) satisfy the above differential equation. Multiplying (32) by $(x-a)^{\alpha}(b-x)^{\beta}$ and rearranging, we have the second differential equation.

Theorem 15. The extended Jacobi functions satisfy the following properties:

(i) $\lim _{v \rightarrow n} F_{v}^{(\alpha, \beta)}(x ; a, b, c)=F_{n}^{(\alpha, \beta)}(x ; a, b, c)$,

(ii) $F_{v}^{(\alpha, \beta)}(-x ; a, b, c)=(-1)^{\nu} F_{v}^{(\alpha, \beta)}(x ;-a,-b, c)$,

(iii) $F_{v}^{(\alpha, \beta)}(a ; a, b, c)=c^{\nu}(a-b)^{\nu}\left(\begin{array}{c}\nu+\alpha \\ \nu\end{array}\right)$,

(iv) $F_{v}^{(\alpha, \beta)}(b ; a, b, c)=(-1)^{v} c^{\nu}(a-b)^{\nu}\left(\begin{array}{c}\nu+\beta \\ v\end{array}\right)$,

(v) $(d / d x) F_{v}^{(\alpha, \beta)}(x ; a, b, c)=c(\nu+\alpha+\beta+1)$ $F_{\gamma-1}^{(\alpha+1, \beta+1)}(x ; a, b, c)$,

(vi) $\left(d^{k} / d x^{k}\right) F_{v}^{(\alpha, \beta)}(x ; a, b, c)=c^{k}(\nu+\alpha+\beta+1)_{k}$ $F_{\nu-k}^{(\alpha+k, \beta+k)}(x ; a, b, c)$ for $k \in \mathbb{N}$.

Proof. Consider the following.

(i) By (20), we have

$$
\begin{aligned}
\lim _{\nu \rightarrow n} F_{\nu}^{(\alpha, \beta)}(x ; a, b, c) & \\
= & \lim _{\nu \rightarrow n} c^{\nu}(a-b)^{\nu}\left(\begin{array}{c}
\nu+\alpha \\
v
\end{array}\right) \\
& \times F\left(-v, \alpha+\beta+\nu+1 ; \alpha+1 ; \frac{a-x}{a-b}\right) \\
= & c^{n}(a-b)^{n}\left(\begin{array}{c}
n+\alpha \\
n
\end{array}\right) F\left(-n, \alpha+\beta+n+1 ; \alpha+1 ; \frac{a-x}{a-b}\right) \\
= & F_{n}^{(\alpha, \beta)}(x ; a, b, c) .
\end{aligned}
$$

(ii) By (13), we get

$$
F_{v}^{(\alpha, \beta)}(-x ; a, b, c)
$$

$$
\begin{aligned}
& =c^{\nu} \sum_{k=0}^{\infty}\left(\begin{array}{c}
\nu+\alpha \\
v-k
\end{array}\right)\left(\begin{array}{c}
\nu+\beta \\
k
\end{array}\right)(-x-a)^{k}(-x-b)^{\nu-k} \\
& =(-1)^{v} F_{v}^{(\alpha, \beta)}(x ;-a,-b, c) .
\end{aligned}
$$

(iii) It is enough to take $x=a$ in (13).

(iv) It proof is enough to take $x=b$ in (27).

(v) Using (20) and differentiating with respect to $x$, the result follows. (vi).

(vi) Repeating $k$ times operation in (v), we obtain 
Corollary 16. As a consequence of Theorem 15(v) and (31), one has the following recurrence relations:

$$
\begin{aligned}
\frac{d}{d x} F_{\nu}^{(\alpha, \beta)}(x ; a, b, c) & \\
=c & \left\{(\beta+\nu) F_{\nu-1}^{(\alpha+1, \beta)}(x ; a, b, c)\right. \\
& \left.+(\alpha+\nu) F_{\nu-1}^{(\alpha, \beta+1)}(x ; a, b, c)\right\}, \\
(\alpha+ & \beta+(\nu+2)) F_{v}^{(\alpha+1, \beta+1)}(x ; a, b, c) \\
= & \left\{(\beta+(\nu+1)) F_{\nu}^{(\alpha+1, \beta)}(x ; a, b, c)\right. \\
& \left.+(\alpha+(\nu+1)) F_{v}^{(\alpha, \beta+1)}(x ; a, b, c)\right\} .
\end{aligned}
$$

Now, we compare some properties of the extended Jacobi functions with extended Jacobi polynomials.

(i) Rodrigues Formula. Consider the following. Extended Jacobi functions:

$$
\begin{aligned}
F_{\nu}^{(\alpha, \beta)}(x ; a, b, c)= & \frac{(-c)^{\nu}}{\Gamma(\nu+1)}(x-a)^{-\alpha}(b-x)^{-\beta} \\
& \times \frac{d^{\nu}}{d x^{\nu}}\left\{(x-a)^{\nu+\alpha}(b-x)^{\nu+\beta}\right\} .
\end{aligned}
$$

Extended Jacobi polynomials:

$$
\begin{aligned}
F_{n}^{(\alpha, \beta)}(x ; a, b, c)= & \frac{(-c)^{n}}{n !}(x-a)^{-\alpha}(b-x)^{-\beta} \\
& \times \frac{d^{n}}{d x^{n}}\left\{(x-a)^{n+\alpha}(b-x)^{n+\beta}\right\} .
\end{aligned}
$$

(ii) Explicit Formula. Consider the following. Extended Jacobi functions:

$$
\begin{aligned}
F_{v}^{(\alpha, \beta)}(x ; a, b, c)=c^{\nu} \sum_{k=0}^{\infty} & \left(\begin{array}{c}
\nu+\alpha \\
\nu-k
\end{array}\right) \\
& \times\left(\begin{array}{c}
\nu+\beta \\
k
\end{array}\right)(x-a)^{k}(x-b)^{\nu-k} .
\end{aligned}
$$

Extended Jacobi polynomials:

$$
\begin{aligned}
F_{v}^{(\alpha, \beta)}(x ; a, b, c)=c^{n} \sum_{k=0}^{\infty} & \left(\begin{array}{c}
n+\alpha \\
n-k
\end{array}\right) \\
& \times\left(\begin{array}{c}
n+\beta \\
k
\end{array}\right)(x-a)^{k}(x-b)^{n-k} .
\end{aligned}
$$

(iii) Hypergeometric Representation. Consider the following. Extended Jacobi functions:

$$
\begin{aligned}
F_{v}^{(\alpha, \beta)}(x ; a, b, c)= & (c)^{\nu}(\mathrm{a}-b)^{\nu}\left(\begin{array}{c}
\nu+\alpha \\
v
\end{array}\right) \\
& \times F\left(-v, \alpha+\beta+\nu+1 ; \alpha+1 ; \frac{a-x}{a-b}\right) .
\end{aligned}
$$

Extended Jacobi polynomials:

$$
\begin{aligned}
F_{n}^{(\alpha, \beta)}(x ; a, b, c)= & (c)^{n}(a-b)^{n}\left(\begin{array}{c}
n+\alpha \\
n
\end{array}\right) \\
& \times F\left(-n, \alpha+\beta+n+1 ; \alpha+1 ; \frac{a-x}{a-b}\right) .
\end{aligned}
$$

(iv) Value at $x=a$. Consider the following. Extended Jacobi functions:

$$
F_{\nu}^{(\alpha, \beta)}(a ; a, b, c)=c^{\nu}(a-b)^{\nu}\left(\begin{array}{c}
v+\alpha \\
v
\end{array}\right)
$$

Extended Jacobi polynomials:

$$
F_{n}^{(\alpha, \beta)}(a ; a, b, c)=c^{n}(a-b)^{n}\left(\begin{array}{c}
n+\alpha \\
n
\end{array}\right)
$$

(v) Value at $x=b$. Consider the following. Extended Jacobi Functions:

$$
F_{v}^{(\alpha, \beta)}(b ; a, b, c)=(-1)^{v} c^{v}(a-b)^{v}\left(\begin{array}{c}
\nu+\beta \\
v
\end{array}\right) \text {. }
$$

Extended Jacobi polynomials:

$$
F_{n}^{(\alpha, \beta)}(b ; a, b, c)=(-c)^{n}(a-b)^{n}\left(\begin{array}{c}
n+\beta \\
n
\end{array}\right)
$$

(vi) Differential Equation. Consider the following. Extended Jacobi functions:

$$
\begin{aligned}
& (x-a)(b-x) Y^{\prime \prime}(x) \\
& \quad+[b(\alpha+1)+a(\beta+1)-x(\alpha+\beta+2)] \\
& \quad \times Y^{\prime}(x)+\nu(\alpha+\beta+\nu+1) Y(x)=0 .
\end{aligned}
$$

Extended Jacobi polynomials:

$$
\begin{aligned}
(x-a) & (b-x) Y^{\prime \prime}(x) \\
& +[b(\alpha+1)+a(\beta+1)-x(\alpha+\beta+2)] \\
& \times Y^{\prime}(x)+n(\alpha+\beta+n+1) Y(x)=0 .
\end{aligned}
$$

(vii) Derivation. Consider the following. Extended Jacobi functions:

$\frac{d}{d x} F_{v}^{(\alpha, \beta)}(x ; a, b, c)=c(\nu+\alpha+\beta+1) F_{\nu-1}^{(\alpha+1, \beta+1)}(x ; a, b, c)$.

Extended Jacobi polynomials:

$\frac{d}{d x} F_{n}^{(\alpha, \beta)}(x ; a, b, c)=\frac{1}{2}(n+\alpha+\beta+1) F_{n-1}^{(\alpha+1, \beta+1)}(x ; a, b, c)$. 


\section{Generalized Extended Jacobi Functions}

In this section, we define a fractional extended Jacobi differential equation and its solution which is the generalized extended Jacobi function.

Definition 17 (see [6]). The fractional hypergeometric differential equation is defined as follows:

$t^{\mu}\left(1-t^{\mu}\right) D^{2 \mu} Y(t)+\left[c-(a+b+1) t^{\mu}\right] D^{\mu}[Y(t)]-a b Y(t)=0$,

where $0<\mu \leq 1$.

Definition 18 (see [6]). The fractional hypergeometric matrix function is defined by

$$
{ }_{2}^{\mu} F_{1}(a, b ; c ; t)=y_{0} t^{p}+\sum_{k=1}^{\infty} y_{0}\left[\prod_{j=0}^{k-1} g_{j}(p) f_{j+1}^{-1}(p)\right] t^{k \mu+p},
$$

where $0<\mu \leq 1$ and

$$
\begin{gathered}
f_{k}(p)=\frac{\Gamma((k \mu+1)+p)}{\Gamma((k-2) \mu+1+p)}+c \frac{\Gamma((k \mu+1)+p)}{\Gamma((k-1) \mu+1+p)}, \\
g_{k}(p)=\frac{\Gamma((k \mu+1)+p)}{\Gamma((k-2) \mu+1+p)}+a b \\
+(a+b+1) \frac{\Gamma((k \mu+1)+p)}{\Gamma((k-1) \mu+1+p)},
\end{gathered}
$$

and the following property

$$
f_{0}(p)=\frac{\Gamma(1+p)}{\Gamma(-2 \mu+1+p)}+c \frac{\Gamma(1+p)}{\Gamma(-\mu+1+p)}=0
$$

holds for $\rho>-1$.

If we take $-v$ instead of $a, a+b+v+1$ instead of $b, a+1$ instead of $c$, and $t \rightarrow(a-t) /(a-b)$ in (53), we obtain the generalized extended Jacobi functions and theirs fractional differential equation.

Definition 19. The fractional extended Jacobi matrix differential equation is defined by

$$
\begin{aligned}
& (-1)^{2 \mu}(a-t)^{\mu}\left[(a-b)^{\mu}-(a-t)^{\mu}\right] D^{2 \mu} Y(t) \\
& +(-1)^{\mu}\left[(a+1)(a-b)^{\mu}-(a+b+2)(a-t)^{\mu}\right] \\
& \quad \times D^{\mu}[Y(t)]+v(a+b+v+1) Y(t)=0
\end{aligned}
$$

where $0<\mu \leq 1 / 2$.
Definition 20. The generalized extended Jacobi functions (GEJFs) are defined by

$$
\begin{aligned}
& { }_{2}^{\mu} F_{1}\left(-v, a+b+(\nu+1) ; a+1 ; \frac{a-t}{a-b}\right) \\
& \quad=y_{0}\left(\frac{a-t}{a-b}\right)^{p}+\sum_{k=1}^{\infty} y_{0}\left[\prod_{j=0}^{k-1} g_{j}(p) f_{j+1}^{-1}(p)\right]\left(\frac{a-t}{a-b}\right)^{p+k \mu},
\end{aligned}
$$

where

$$
\begin{gathered}
f_{k}(p)=\frac{\Gamma((k \mu+1)+p)}{\Gamma((k-2) \mu+1+p)} \\
+(a+1) \frac{\Gamma((k \mu+1)+p)}{\Gamma((k-1) \mu+1+p)}, \\
g_{k}(p)=\frac{\Gamma((k \mu+1)+p)}{\Gamma((k-2) \mu+1+p)}-v(a+b+(\nu+1)) \\
+(a+b+2) \frac{\Gamma((k \mu+1)+p)}{\Gamma((k-1) \mu+1+p)}
\end{gathered}
$$

and the equation

$$
f_{0}(p)=\frac{\Gamma(1+p)}{\Gamma(-2 \mu+1+p)}+(a+1) \frac{\Gamma(1+p)}{\Gamma(-\mu+1+p)}=0
$$

holds for $\rho>-1$.

Theorem 21. The generalized extended Jacobi function is a solution of (57).

Proof. Using Definitions 17 and 18 and (5), the theorem can be proved.

\section{References}

[1] K. S. Miller and B. Ross, An Introduction to the Fractional Calculus and Fractional Differential Equations, John Wiley \& Sons, New York, NY, USA, 1993.

[2] K. B. Oldham and J. Spanier, The Fractional Calculus; Theory and Applications of Differentiation and Integration to Arbitrary Order, Mathematics in Science and Engineering, Academic Press, New York, NY, USA, 1974.

[3] I. Podlubny, Fractional Differential Equations, Mathematics in Science and Engineering, Academic Press, San Diego, Calif, USA, 1999.

[4] G. Jumarie, "Fractional Euler'sintegral of first and second kinds. Application to fractional Hermite's polynomials and to probability density of fractional orders," Journal of Applied Mathematics \& Informatics, vol. 28, no. 1-2, pp. 257-273, 2010.

[5] I. Fujiwara, "A unified presentation of classical orthogonal polynomials," Mathematica Japonica, vol. 11, pp. 133-148, 1966.

[6] S. P. Mirevski, L. Boyadjiev, and R. Scherer, "On the RiemannLiouville fractional calculus, $g$-Jacobi functions and $F$-Gauss functions," Applied Mathematics and Computation, vol. 187, no. 1, pp. 315-325, 2007. 


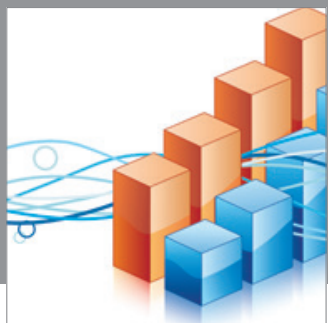

Advances in

Operations Research

mansans

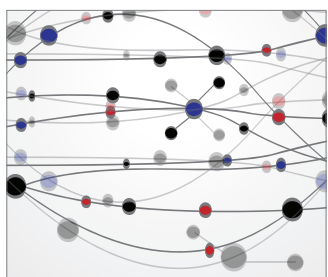

The Scientific World Journal
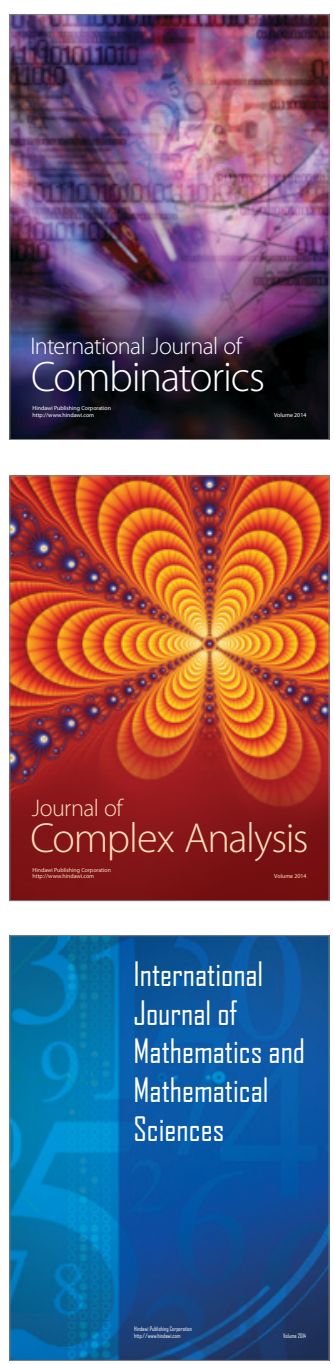
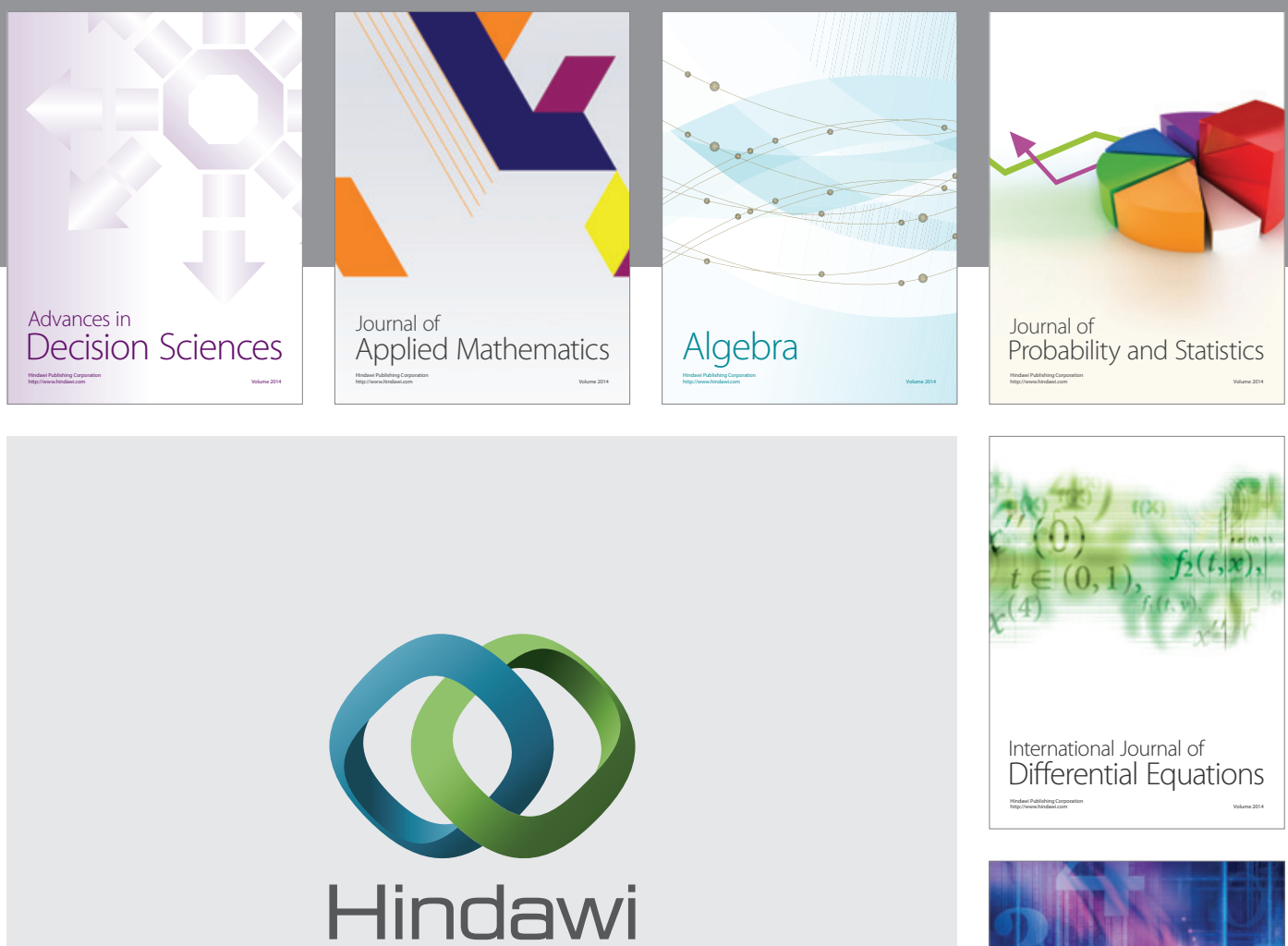

Submit your manuscripts at http://www.hindawi.com
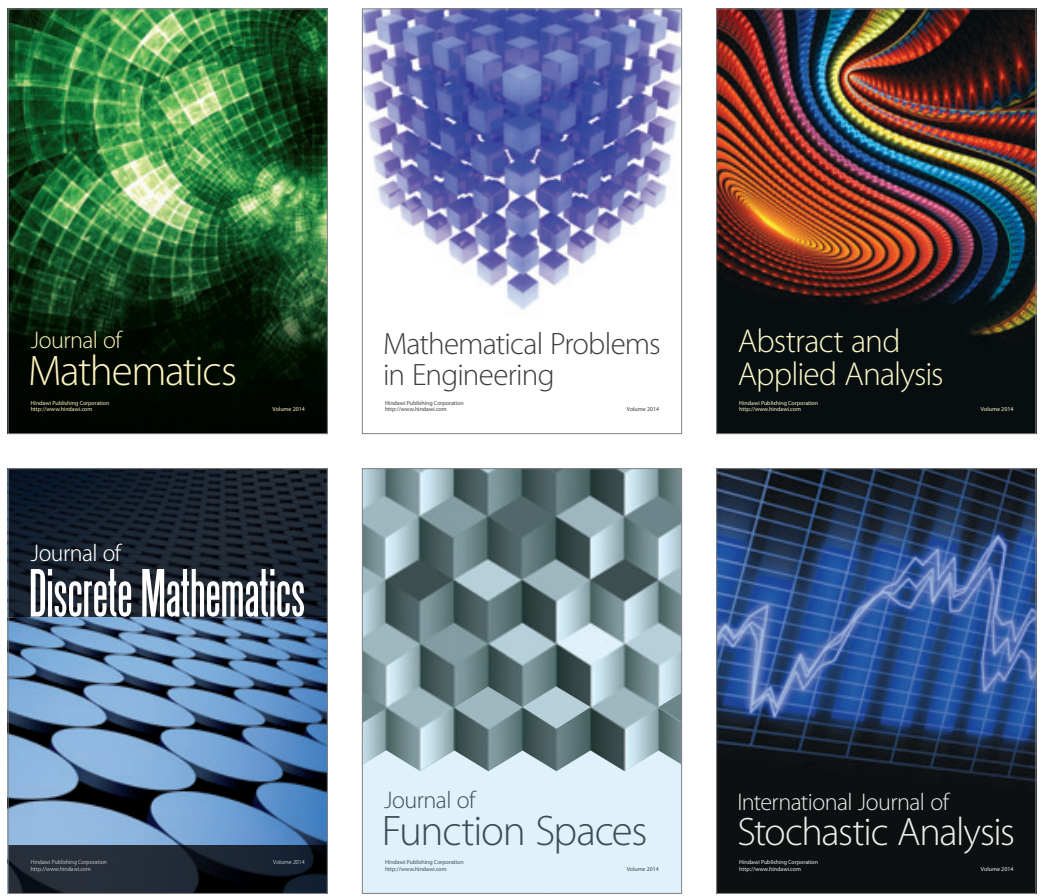

Journal of

Function Spaces

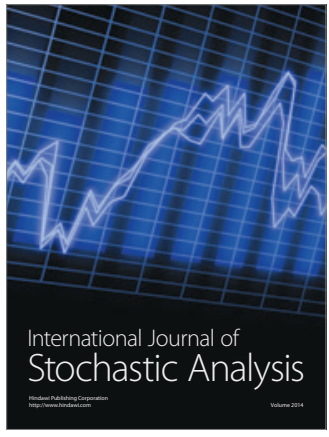

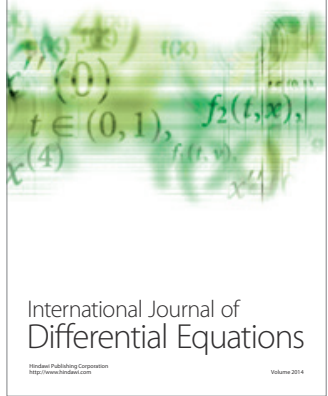
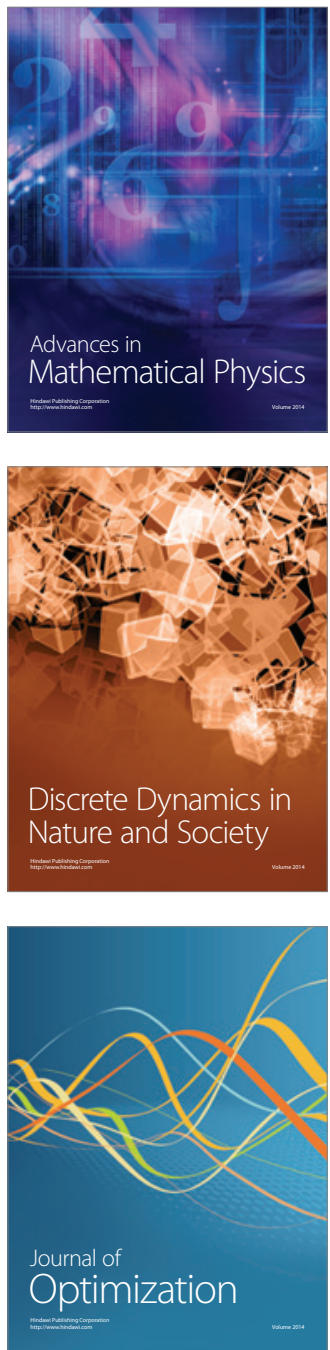